\title{
Evaluación del impacto económico de la vacuna antirrotavírica en Venezuela
}

\author{
Dagna Constenla, ${ }^{1}$ Irene Pérez-Schael, ${ }^{2}$ Richard D. Rheingans, ${ }^{1}$ \\ Lynn Antil, ${ }^{1}$ Hans Salas ${ }^{3}$ y Juan Pablo Yarzábal ${ }^{4}$
}

Forma de citar Constenla D, Pérez-Schael I, Rheingans RD, Antil L, Salas H, Yarzábal JP. Evaluación del impacto económico de la vacuna antirrotavírica en Venezuela. Rev Panam Salud Publica. 2006;20(4):213-22.

RESUMEN Objetivo. El objetivo de este estudio fue evaluar el costo de la atención médica de la gastroenteritis por rotavirus y la relación costo-efectividad de la vacuna antirrotavírica en la población venezolana menor de 5 años de edad.

Métodos. Se utilizó un modelo económico que integra la información epidemiológica, la eficacia de la vacuna y los costos de atención médica de la gastroenteritis por rotavirus, desde la perspectiva de la sociedad. Para determinar la efectividad de la vacuna, se estimó el número de casos de hospitalización, de consultas médicas y de muertes evitados después de su administración. La relación costo-efectividad de la vacuna se evaluó partiendo del número de años de vida ajustados por discapacidad (AVAD) y de casos evitados.

Resultados. En Venezuela, los servicios de salud invierten, aproximadamente, 4,2 millones de dólares estadounidenses (US\$) por año para cubrir los costos de atención médica causados por el rotavirus. Un programa de vacunación antirrotavírica evitaría aproximadamente el $52 \%$ (186) de las muertes, el 54\% (7 232) de las hospitalizaciones y el 50\% (55 168) de las consultas ambulatorias durante los primeros cinco años de vida, en una cohorte vacunada. Para un precio estimado de US\$24 por régimen de vacuna, se genera una relación costo-efectividad de US\$1352 por AVAD. Conclusiones. Los resultados de este estudio apuntan a que la vacunación antirrotavírica es una estrategia costoefectiva en la prevención de la gastroenteritis por rotavirus en Venezuela, ya que puede evitar muertes y años de vida ajustados por discapacidad en la población menor de cinco años de edad.

Palabras clave Rotavirus, vacunación, análisis económico, prevención, gastroenteritis, Venezuela.

En Venezuela existe un interés creciente por los estudios de impacto económico y de costo-efectividad, y se reconoce que estos últimos son esenciales

1 Department of Global Health, Rollins School of Public Health, Emory University, Atlanta, Georgia, Estados Unidos de América.

2 Instituto de Biomedicina-Fuvesin, Universidad Central de Venezuela, Ministerio de Salud, Caracas, Venezuela.

3 Instituto Nacional de Higiene "Rafael Rangel", Universidad Central de Venezuela, Caracas, Venezuela.

4 GlaxoSmithKline Biologicals, Caracas, Venezuela. para la toma de decisiones en materia de salud. La principal dificultad para realizar estos estudios es la disponibilidad de la información necesaria para alimentar el modelo de análisis. Cuando no se dispone de estimaciones detalladas y confiables sobre los indicadores epidemiológicos y los costos del modelo, es muy difícil llegar a conclusiones que satisfagan a los usuarios.

La gastroenteritis por rotavirus constituye una de las principales cau- sas de morbilidad y mortalidad en el mundo. Por esta razón, se despliegan numerosos esfuerzos para desarrollar una vacuna que pueda prevenir la enfermedad grave causada por este virus. En Venezuela, la enfermedad por rotavirus ha sido muy estudiada durante los últimos 30 años, desde el punto de vista virológico, clínico, epidemiológico y profiláctico, lo que permite la realización de estudios de costo-efectividad (1-4). 
El impacto de la infección por rotavirus como causa de gastroenteritis fue estudiado recientemente en América Latina (5-8) y, en especial, en Venezuela $(1-4,8)$. Un estudio de vigilancia epidemiológica de la diarrea aguda, realizado en el hospital público Ciudad Hospitalaria Dr. Enrique Tejera (CHET), en Valencia (Estado Carabobo, Venezuela), permitió estimar la importancia del rotavirus como causa de consulta médica y de hospitalización de niños menores de 5 años de edad, durante un período de cinco años (1). En dicho estudio, el rotavirus originó $23 \%$ de todas las consultas por diarrea en el hospital $(20 \%$ entre los pacientes ambulatorios y $31 \%$ entre los pacientes hospitalizados).

En otro estudio anterior, realizado en la ciudad de Caracas, la capital del país, se estimó la importancia clínica del rotavirus en la población menor de 3 años de edad (3). En dicho estudio, el rotavirus causó 35\% de las consultas médicas por diarrea, con una frecuencia de $24 \%$ entre los pacientes ambulatorios y de $76 \%$ entre los pacientes hospitalizados. La prevalencia de la infección por rotavirus fue independiente del estrato socioeconómico. Sin embargo, el riesgo de deshidratación asociado fue mayor en los estratos socioeconómicos más bajos. El 85\% de los episodios de gastroenteritis por rotavirus ocurrieron durante el primer año de vida. Estos estudios evidencian un impacto importante del rotavirus en la morbilidad en este grupo de edad en Venezuela.

La información epidemiológica disponible sobre la infección por rotavirus, reciente y de calidad, y los datos demográficos del país, asociados a la información recolectada sobre los recursos médico-hospitalarios y sus costos, y la perspectiva muy cercana de una vacuna que prevenga sus manifestaciones clínicas más graves, permiten la evaluación correcta de la relación costoefectividad de la vacuna antirrotavírica.

Este estudio forma parte de un proyecto más amplio que se llevó a cabo en diferentes países de la Región, en el cual se consultaron diversas fuentes de información con cobertura en Venezuela. En consecuencia, los resultados que se presentan en este artículo pue- den diferir de los presentados en el análisis regional $(9,10)$.

\section{MÉTODOS}

\section{Diseño General}

En este estudio, se utilizó un modelo económico fundamentado en los principios del análisis de costo-efectividad, con el objeto de evaluar la aplicación de un programa de vacunación antirrotavírica y compararla con la estrategia sin programa de vacunación y con el tratamiento habitual de la enfermedad por rotavirus en la práctica clínica estándar, en la población infantil venezolana. La cohorte teórica anual de nacidos vivos en Venezuela utilizada en el modelo económico fue de 582 000, correspondiente al año 2003 (11).

En este análisis, para calcular la incidencia de la enfermedad por rotavirus y los costos hospitalarios relacionados, se consideraron las estadísticas nacionales y los datos provenientes de los estudios publicados en las poblaciones atendidas en el sector público, que representan $80 \%$ de la población infantil venezolana (11). Se utilizó como indicador principal la variable "años de vida ajustados por discapacidad" (AVAD). Esta variable tiene en cuenta el impacto de una determinada enfermedad asociado a la pérdida de años de vida (en caso de muerte relacionada con el evento) y a la disminución de la calidad de vida (período de tiempo que el paciente vive con una discapacidad relacionada con el evento). En el caso de la infección por rotavirus, que no suele dejar secuelas, el número de AVAD deriva de los casos de muerte prematura que se relacionan con la enfermedad.

El análisis de costo-efectividad se realizó desde la perspectiva de la sociedad, por lo que se incluyeron los costos médicos directos (medicamentos, estancia hospitalaria, consultas ambulatorias, pruebas diagnósticas y otros procedimientos complementarios), los costos directos no médicos (gastos de transporte) y los costos indirectos (pérdida de productividad por baja laboral o ausencia del trabajo de los padres de niños enfermos). Para estimar los costos de cada intervención, se utilizó el método de costos por actividades, mediante el cual se identifican los insumos y los procesos de atención y se estiman los costos medios por caso. Para el cálculo de las incidencias y de los costos se tuvieron en cuenta los datos de los sistemas público y privado.

Los costos y los beneficios del programa de vacunación se actualizaron respecto al año 2003 utilizando una tasa de descuento de 3\%. Todos los datos monetarios se expresaron en dólares estadounidenses (US\$) de 2003, aplicando una tasa de cambio de 1 606,96 bolívares por dólar (12).

\section{Cálculo de los indicadores \\ de morbilidad y de la tasa de mortalidad por rotavirus}

En este estudio, se consideró "caso de hospitalización" todo episodio de infección por rotavirus que hubiese motivado una hospitalización o una permanencia en la sala de observación del hospital durante una noche o más. La incidencia acumulada se determinó a partir del número de episodios de infección por rotavirus ocurridos en la población menor de 5 años y el tamaño de la cohorte de nacidos vivos. Los cálculos de los indicadores de morbilidad se basaron en las estimaciones realizadas en un estudio recientemente publicado (1).

En dicho estudio, el rotavirus ocasionó 39127 ingresos hospitalarios de menores de 5 años en una cohorte de 561502 nacidos vivos (cuadro 1). Según los datos del sistema de vigilancia, en Valencia 33\% de los pacientes ingresados permanecieron una o más noches en el hospital (Irene PérezSchael, comunicación personal, 2004), por lo que se consideró que el rotavirus había ocasionado 12912 casos de hospitalización entre los menores de 5 años. En consecuencia, el riesgo acumulado de hospitalización por rotavirus en niños menores de 5 años es 0,023 (12 912/561 502). Esto representa una tasa acumulada de 23 hospitalizaciones por rotavirus por cada 1000 niños menores de 5 años. 
CUADRO 1. Resumen de los datos e indicadores utilizados para obtener las

\begin{tabular}{lr}
\hline \multicolumn{1}{c}{ Dato o indicador } & \multicolumn{1}{c}{ Valor } \\
\hline Tamaño de la cohorte de nacidos vivos & 561502 \\
Número de ingresos hospitalarios por rotavirus & 39127 \\
Número de casos de hospitalización (estadía $\geq 1$ noche) & 12912 \\
Riesgo acumulado de hospitalización por rotavirus & 0,023 \\
Número total de consultas por rotavirus & 118567 \\
Número de consultas ambulatorias por rotavirus & 105655 \\
Riesgo acumulado de atención ambulatoria por rotavirus & 0,19 \\
Riesgo acumulado de muerte por rotavirus & 0,00063 \\
\hline
\end{tabular}

a Los cálculos se basaron en las estimaciones de Salinas et al., 2004 (1). variables aplicadas en el modelo ${ }^{a}$

en un $77,5 \%$, basándose en un promedio entre la prevención de episodios graves $(85 \%)$ (19) y de episodios de cualquier gravedad (70\%) (20). Se utilizó un valor de cobertura del $68 \%$, correspondiente a la cobertura alcanzada actualmente por el Ministerio de Salud en la administración de la tercera dosis de la vacuna combinada contra la difteria, la tos ferina y el tétanos (vacuna DPT) (21) (cuadro 2).

\section{Costo del programa de vacunación}

El costo del programa de vacunación deriva del costo de administración (US\$ 1 por régimen de vacunación), del precio estimado de la vacuna (US\$ 12 por dosis), del número de dosis administradas según la cobertura y de la pérdida por desperdicio, que se anticipa en un $10 \%$. La vacuna contra el rotavirus se administraría dentro del Programa Ampliado de Inmunizaciones (PAI) actual, por lo que no se prevén mayores costos incrementales. El precio estimado de las dos dosis de vacuna es de US\$ 24 (cuadro 2).

(105 655/561 502), lo que representa una tasa acumulada de 190 casos por cada 1000 niños menores de 5 años (cuadro 1).

Para el cálculo de la mortalidad, se partió del número de muertes por infecciones intestinales (CIE-10: A00A09) de niños venezolanos menores de 5 años, el cual ascendió a 1147 en 2001 (13), lo que representa una tasa de mortalidad por infección intestinal de 200 por 100000 niños menores de 5 años. Partiendo de la mortalidad por rotavirus, estimada en un $31 \%$ (2), se calculó que de las 1147 muertes por diarrea, 356 se debían al rotavirus (2). Por lo tanto, se considera que la tasa acumulada de mortalidad por rotavirus en menores de 5 años es 0,00063 (356/561 502), lo que representa 0,63 muertes por 1000 niños menores de 5 años (cuadro 1).

\section{Años de vida ajustados por discapacidad (AVAD)}

La efectividad medida en términos de AVAD se calculó a partir de las es- peranzas de vida al nacer y al cumplir el primer año de vida, las cuales, según estimaciones del Sistema Estadístico y de Informática de la Organización Mundial de la Salud (OMS), corresponden a 71 y 77 años, respectivamente (14) (cuadro 2). El cálculo del número de AVAD se basó en la información sobre la mortalidad por rotavirus en ocho grupos de edad (0-2 meses, 3-5 meses, 6-8 meses, 9-11 meses, 12-23 meses, 24-35 meses, 3647 meses y 48-59 meses) en que se ha dividido la población susceptible de vacunación $(3-6,15)$, en un promedio ponderado de los años de vida ajustados por discapacidad proveniente del estudio global de carga de enfermedad (16) y en las guías de costo-efectividad de la OMS (17). Asimismo se consideró que la enfermedad por rotavirus tiene una duración estimada de seis días (18).

\section{Eficacia y cobertura de la vacuna}

Se consideró que la eficacia vacunal era de un 84,7\% (IC95\%: 71,7\%-92,4\%) en la prevención de episodios graves de gastroenteritis por rotavirus y de un 85\% (IC95\%: 69,6\%-93,5\%) en la prevención de episodios de gastroenteritis asociados con el rotavirus que requieren hospitalización (19). Se supuso que la vacuna tendría una eficacia de un $85 \%$ para evitar muertes por rotavirus. La eficacia en la prevención de casos de gastroenteritis que motivan consultas ambulatorias se calculó

\section{Estimación de los costos médicos directos}

Los costos de hospitalización se calcularon utilizando datos de los departamentos de contabilidad de dos hospitales privados y de la Secretaría de Salud del Estado Carabobo o la Fundación Instituto Carabobeño para la Salud (INSALUD). El costo medio de hospitalización fue de US\$19,91 por día. Según datos de un estudio reciente de vigilancia hospitalaria de la gastroenteritis por rotavirus (7), se estimó que la duración media de la estancia en el hospital es de 4,1 días. $\mathrm{Al}$ multiplicar estos dos valores, se obtuvo un costo total de hospitalización por paciente de US\$ 81,63 (cuadro 3).

En el sector público, el costo medio de las consultas médicas fue de US\$ 5,48 por consulta, según se calculó a partir de datos del mismo estudio de vigilancia hospitalaria (7) y del promedio ponderado de consultas médicas pediátricas y de consultas en servicios 


\section{CUADRO 2. Variables aplicadas en el modelo}



a Se incluyen los costos de hospitalización, de las consultas medicas, de los medicamentos, de las pruebas diagnósticas y de los procedimientos complementarios. Los valores se basan en un promedio de los datos de los sectores público y privado.

b Los valores están expresados en dólares estadounidenses (US\$) de 2003.

\section{CUADRO 3. Costo de la gastroenteritis por rotavirus ${ }^{\mathrm{a}, \mathrm{b}}$}

\begin{tabular}{|c|c|c|c|c|}
\hline \multirow[b]{2}{*}{ Variable } & \multicolumn{2}{|c|}{$\begin{array}{c}\text { Grupo de pacientes } \\
\text { hospitalizados }(n=196)\end{array}$} & \multicolumn{2}{|c|}{$\begin{array}{l}\text { Grupo de pacientes } \\
\text { ambulatorios }(n=91)\end{array}$} \\
\hline & Promedio & Intervalo & Promedio & Intervalo \\
\hline \multicolumn{5}{|l|}{ Costo diario de la estancia/cama o } \\
\hline costo de la consulta & US\$ 19,91 & & US\$ 5,48 & \\
\hline Duración de la estancia & 4,10 días & & & \\
\hline Pruebas diagnósticas & US\$ 20,86 & US\$ $(14,00-21,30)$ & US $\$ 11,00$ & US\$ $(6,66-14,00)$ \\
\hline Total por paciente & US\$ 124,94 & & US\$ 23,27 & \\
\hline \multicolumn{5}{|l|}{ Costos directos no médicos } \\
\hline Gastos de transporte & US\$ 4,91 & & US\$ 1,55 & \\
\hline Total por paciente & US\$ 4,91 & & US\$ 1,55 & \\
\hline \multicolumn{5}{|l|}{ Costos indirectos } \\
\hline Pérdida de productividad notificada & $45,5 \%$ & & $28,6 \%$ & \\
\hline
\end{tabular}

a Estos valores derivan de los datos de en un estudio de vigilancia hospitalaria de la gastroenteritis por rotavirus (7).

b Los valores están expresados en dólares estadounidenses (US\$) de 2003. 
de urgencia y de rehidratación, y de la información proveniente de entrevistas realizadas a médicos y pediatras (cuadro 3). Según el sistema de vigilancia, el 7\% de los niños con diarrea que llegan al hospital público se ingresa, el $15 \%$ se trata en observación y el $78 \%$ se trata ambulatoriamente (1, Irene Pérez-Schael, comunicación personal, 2004).

El número de pruebas de laboratorio y de procedimientos complementarios del tratamiento de la gastroenteritis también se estimó a partir de datos del estudio de vigilancia hospitalaria citado (7). Los costos de estas pruebas y de estos procedimientos complementarios se calcularon utilizando los datos de contabilidad de los hospitales anteriormente mencionados. El costo medio de las pruebas de laboratorio y de los procedimientos complementarios fue de US\$ 8,34 en el caso de los pacientes hospitalizados y de US\$ 7,33 en el de los pacientes ambulatorios. Este valor incluye los análisis de sangre, orina y heces. A partir de los datos del estudio de vigilancia hospitalaria de la gastroenteritis, se estimó un promedio de 2,5 pruebas de laboratorio por paciente hospitalizado y 1,5 pruebas de laboratorio por paciente ambulatorio, lo que corresponde a un costo total de pruebas de laboratorio de US\$20,86 por paciente hospitalizado y US\$11,00 por paciente ambulatorio (cuadro 3 ).

Las dosis y la duración del tratamiento administrado a los pacientes con gastroenteritis también se estimaron a partir de los resultados del estudio mencionado de vigilancia hospitalaria de la gastroenteritis (7). Para estimar el costo de los medicamentos por paciente tratado, se calculó el costo de un tratamiento farmacológico completo según los esquemas terapéuticos aplicados en cada situación, basándose en el costo unitario de cada medicamento - de acuerdo con los precios de mercado propuestos por los fabricantes en 2003 a las instituciones de salud mencionadas anteriormente- - en el número de dosis administradas. Se calculó así un costo medio de medicamentos de US\$14,03 por paciente hospitalizado y de US\$2,72 por paciente ambulatorio. Este costo incluye el costo de la administración de sales de rehidratación oral y de soluciones de rehidratación intravenosa. Según los datos del estudio de vigilancia hospitalaria (7), se estimó un promedio de 1,6 sesiones de rehidratación intravenosa por paciente hospitalizado y 2,5 sobres de sales de rehidratación oral por paciente ambulatorio. Basándose en esta información, se calculó que el costo total de medicamentos fue de US\$22,45 por paciente hospitalizado y de US\$ 6,79 por paciente ambulatorio (cuadro 3).

\section{Estimación de los costos directos no médicos}

Los costos de transporte de los niños con infección por rotavirus y de los familiares que los cuidan se estimaron utilizando los datos del estudio de vigilancia hospitalaria de la gastroenteritis mencionado (7). En este estudio, comunicaron gastos de transporte los padres o los familiares de aproximadamente un 58,2\% de los niños hospitalizados y un 52,4\% de los niños tratados ambulatoriamente. A partir de esta información, se calculó un promedio de 2,87 viajes realizados en el grupo de pacientes hospitalizados y de 1,48 viajes realizados en el grupo de pacientes ambulatorios. El costo medio directo no médico se estimó a partir del número medio de viajes realizados y del costo medio de cada viaje. Así, el costo total estimado de transporte de los niños infectados por el rotavirus y de sus familiares fue de US\$ 4,91 por niño hospitalizado y US\$ 1,55 por niño tratado ambulatoriamente (cuadro 3 ).

\section{Estimación de los costos indirectos}

Los costos indirectos relacionados con la pérdida de productividad por bajas laborales o ausencia en el trabajo de los padres o familiares que cuidaban a los niños enfermos se calcularon basándose en la información recogida en el estudio de vigilancia hospitalaria de la gastroenteritis mencionado (7). Comunicaron una pérdida salarial al- rededor del $45,5 \%$ y del $28,6 \%$ de los padres y familiares de pacientes hospitalizados y pacientes ambulatorios, respectivamente. A partir de esta información, se estimó una pérdida media de trabajo de 18,2 horas por familia en el grupo de niños hospitalizados y de 13,7 horas en el grupo de niños tratados ambulatoriamente. El costo medio indirecto de esta pérdida se calculó a partir del salario medio por hora de las mujeres, que es de US\$1,05, según estimaciones de la Encuesta de hogares (22). Así, el costo indirecto total estimado fue de US\$ 8,85 por paciente hospitalizado y de US\$ 4,10 por paciente ambulatorio (cuadro 3 ).

\section{Análisis de sensibilidad}

Se realizó un análisis de sensibilidad con una variable para evaluar el impacto que tendrían algunos cambios en los principales parámetros del modelo. Concretamente, se consideró el efecto de una variación del $20 \%$ de las siguientes variables: indicadores de morbilidad y mortalidad por rotavirus, eficacia de la vacuna para combatir la mortalidad y la morbilidad por rotavirus, costos del tratamiento actual de la gastroenteritis por rotavirus y precio estimado de la vacuna.

\section{RESULTADOS}

Los resultados de esta investigación se presentan en dos partes. En la primera, se destacan los eventos (hospitalizaciones, consultas médicas, muertes y AVAD) y los costos evitados tras la eventual incorporación de la vacuna contra el rotavirus. En la segunda, se exponen los resultados de la evaluación de costo-efectividad de la vacuna antirrotavírica.

\section{Eventos estimados con programa de vacunación y sin programa de vacunación}

En el cuadro 4, se presentan los números de eventos estimados para una cohorte de nacidos vivos (582 000 
CUADRO 4. Número de eventos estimados en una cohorte de nacidos vivos durante los primeros 5 años de vida con vacunación y sin vacunación

\begin{tabular}{|c|c|c|}
\hline & $\begin{array}{l}\text { Número total } \\
\text { de eventos }\end{array}$ & $\begin{array}{l}\text { Número de eventos } \\
\text { por } 1000 \text { niños }\end{array}$ \\
\hline \multicolumn{3}{|c|}{ Situación actual (en ausencia de programa de vacunación) } \\
\hline Hospitalizaciones & 13502 & 23 \\
\hline Consultas médicas ambulatorias & 110580 & 190 \\
\hline Muertes & 361 & 0,6 \\
\hline $\mathrm{AVAD}^{\mathrm{b}}$ & 12315 & 21 \\
\hline \multicolumn{3}{|l|}{ Situación con programa de vacunación } \\
\hline Hospitalizaciones & 6270 & 11 \\
\hline Consultas médicas ambulatorias & 55412 & 95 \\
\hline Muertes & 175 & 0,3 \\
\hline$A V A D^{b}$ & 5967 & 10 \\
\hline \multicolumn{3}{|c|}{ Beneficio de la vacunación (eventos evitados) ${ }^{c}$} \\
\hline Hospitalizaciones & 7232 & 12 \\
\hline Consultas médicas ambulatorias & 55168 & 95 \\
\hline Muertes & 186 & 0,3 \\
\hline$A \bigvee A D^{b}$ & 6348 & 11 \\
\hline \multicolumn{3}{|c|}{$\begin{array}{l}\text { a El cálculo del número total de se basó en la incidencia o los riesgos calculados a partir de los artículos de Salinas et al., } \\
2004 \text { (1), y Parashar et al., } 2003 \text { (2), extrapolados a la cohorte de } 582.000 \text { nacidos vivos. } \\
\text { b } \text { AVAD = años de vida ajustados por discapacidad. Se utilizó una tasa de descuento del } 3 \% \text {. } \\
\text { c Beneficio de la vacunación basado en la efectividad de la vacuna, que incorpora información sobre la eficacia y la cobertura } \\
\text { de la vacuna. }\end{array}$} \\
\hline
\end{tabular}

CUADRO 5. Carga económica estimada del rotavirus y beneficios de la vacunación en Venezuela

\begin{tabular}{|c|c|c|}
\hline & $\begin{array}{l}\text { Costo total } \\
\text { (US\$) }\end{array}$ & $\begin{array}{c}\text { Costo medio por niñoa } \\
\text { (US\$) }\end{array}$ \\
\hline \multicolumn{3}{|c|}{ Situación actual (sin programa de vacunación) } \\
\hline \multicolumn{3}{|l|}{ Costos directos médicos } \\
\hline Hospitalizaciones & 1662231 & 2,86 \\
\hline Consultas médicas ambulatorias & 2513250 & 4,32 \\
\hline Total & 4175481 & 7,18 \\
\hline \multicolumn{3}{|l|}{ Costos totales ${ }^{b}$} \\
\hline Hospitalizaciones & 1844136 & 3,17 \\
\hline Consultas médicas ambulatorias & 3123524 & 5,37 \\
\hline Total & 4967660 & 8,54 \\
\hline \multicolumn{3}{|l|}{ Situación con programa de vacunación } \\
\hline \multicolumn{3}{|l|}{ Costos directos médicos } \\
\hline Hospitalizaciones & 772368 & 1,33 \\
\hline Consultas médicas ambulatorias & 1260024 & 2,16 \\
\hline Total & 2032392 & 3,49 \\
\hline \multicolumn{3}{|l|}{ Costos totales ${ }^{b}$} \\
\hline Hospitalizaciones & 856891 & 1,47 \\
\hline Consultas médicas ambulatorias & 1565986 & 2,69 \\
\hline Total & 2422877 & 4,16 \\
\hline \multicolumn{3}{|c|}{ Beneficio de la vacunación (costos evitados) } \\
\hline \multicolumn{3}{|l|}{ Costos directos médicos } \\
\hline Hospitalizaciones & 889863 & \\
\hline Consultas médicas ambulatorias & 1253226 & \\
\hline Total & 2143089 & \\
\hline \multicolumn{3}{|l|}{ Costos totales ${ }^{b}$} \\
\hline Hospitalizaciones & 987245 & \\
\hline Consultas médicas ambulatorias & 1557538 & \\
\hline Total & 2544783 & \\
\hline
\end{tabular}

\footnotetext{
a Los valores están expresados en dólares estadounidenses (US\$) de 2003.
}

b Incluye los costos directos médicos, los costos directos no médicos y los costos indirectos. niños) durante los primeros 5 años de vida en dos situaciones: con vacunación y sin vacunación. En Venezuela, se estima que el rotavirus origina en esta cohorte vacunada alrededor de 110500 consultas médicas, 13500 hospitalizaciones y 361 muertes durante los primeros 5 años de vida. La administración de la vacuna antirrotavírica evitaría 55168 consultas médicas, 7232 hospitalizaciones y un total de 186 muertes.

\section{Costos de atención médica calculados y beneficios de la vacunación}

En ausencia de un programa de vacunación, la gastroenteritis por rotavirus tiene un costo total anual de US\$ 4,2 millones para cubrir gastos de atención médica (cuadro 5). El programa de vacunación supondría una economía de US\$2,1 millones anuales para el sistema de salud, es decir, una disminución del $51 \%$ de los costos directos médicos totales ocasionados por el rotavirus.

\section{Costos directos no médicos y costos indirectos calculados y beneficios de la vacunación}

Se gastan anualmente alrededor de US\$ 232330 en transporte como consecuencia de la gastroenteritis por rotavirus. Los costos indirectos asociados a la pérdida de productividad por bajas laborales o ausencia en el trabajo ascienden a US\$ 559 850. El costo total de la gastroenteritis por rotavirus es ligeramente inferior a US\$ 5 millones (incluidos los costos de transporte y la pérdida de trabajo) (cuadro 5). Si se tienen en cuenta los costos no médicos y los costos indirectos, el programa de vacunación supone una economía de US\$2,5 millones para la sociedad.

\section{Relación costo-efectividad potencial de la vacuna antirrotavírica}

En el cuadro 6 se presentan los beneficios potenciales de la vacuna contra 
CUADRO 6. Estimaciones de costos, beneficios netos y costo-efectividad de la vacuna antirrotavírica en Venezuela

\begin{tabular}{|c|c|}
\hline & $\begin{array}{l}\text { Valor } \\
(\text { US\$) }\end{array}$ \\
\hline \multicolumn{2}{|l|}{ Costo del programa de vacunación ${ }^{b}$} \\
\hline Administración de la vacuna & 395760 \\
\hline Adquisición de la vacuna (US\$24/régimen) & 10448064 \\
\hline Total & 10843824 \\
\hline Costos evitados & 2143089 \\
\hline Costo directo médico neto & 8700735 \\
\hline \multicolumn{2}{|l|}{ Costo-efectividad incremental } \\
\hline Costo por $A V A D^{c}$ evitado & 1371 \\
\hline Costo por muerte evitada & 46698 \\
\hline Costo por hospitalización evitada & 1203 \\
\hline Costo por visita médica ambulatoria evitada & 154 \\
\hline \multicolumn{2}{|c|}{$\begin{array}{l}\text { a Los valores están expresados en dólares estadounidenses (US\$) de 2003. Para calcular el costo } \\
\text { de la intervención, el costo directo médico neto y la relación costo-efectividad incremental, se con- } \\
\text { sideró el precio actual de la vacuna de US\$24 por régimen. } \\
\text { b El costo de vacunación se calcula basándose en la estimación de cobertura de la vacuna y en la } \\
\text { pérdida de desperdicio del programa, que se anticipa en un } 10 \% \text {. } \\
\text { c AVAD = años de vida ajustados por discapacidad. }\end{array}$} \\
\hline
\end{tabular}

el rotavirus desde la perspectiva del servicio de salud, expresados como costos médicos netos y como relaciones de costo-efectividad incremental. En las condiciones en que se basaron los cálculos, el costo directo médico neto de la vacunación de esta cohorte de nacidos vivos en Venezuela se estimó en US\$ 8,7 millones. Desde el punto de vista del sistema de salud, esto proporcionaría una relación costoefectividad de US\$ 1371 por AVAD evitado y US\$ 46698 por muerte evitada. Desde el punto de vista de la sociedad, la relación costo-efectividad potencial de la vacuna antirrotavírica es de US\$ 1352 por AVAD.

A partir de los resultados del presente estudio, se puede estimar el precio de equilibrio de la intervención, que permite generar ahorros notables sin ocasionar gastos adicionales para el servicio de salud o la sociedad. El precio de la vacuna no podrá exceder US\$ 4,01 por régimen para conseguir que el programa de vacunación origine un ahorro para el sistema de salud.

\section{Análisis de sensibilidad}

Como puede observarse en el cuadro 7 , los resultados del estudio de costos dependieron de la incidencia de in- fección por rotavirus y de los costos médicos ocasionados por esta enfermedad, ya que una variación del $20 \%$ en los valores de estas variables se asoció con una desviación del 3\% al 12\% en el costo médico por niño. Al disminuir la incidencia o el costo sanitario, la carga financiera atribuible al rotavirus también disminuye. Los resultados del estudio de costo-efectividad dependieron de las siguientes variables: mortalidad por rotavirus, eficacia de la vacuna para evitar muertes por rotavirus y precio de la vacuna. Una variación del $20 \%$ en los valores de estas variables se asoció con una variación del $15 \%$ al $40 \%$ en la relación costo-efectividad de la vacuna. Los aumentos en la mortalidad y la eficacia de la vacuna para evitar muertes generaron la menor relación costo-efectividad incremental.

\section{DISCUSIÓN}

En Venezuela, la gastroenteritis grave por rotavirus representa una carga de morbilidad importante, ya que el rotavirus causa un número considerable de diarreas que requieren hospitalización o tratamiento ambulatorio en los servicios de urgencias de los hospitales (1-3). En este estudio, se estimó que un programa de vacunación antirrotavírica evitaría alrededor de 186 muertes, 7232 hospitalizaciones y 55168 consultas ambulatorias durante los primeros 5 años de vida, en la cohorte considerada.

En cuanto a la carga económica de la infección por rotavirus, los resultados de esta investigación indican que, por cada 1000 niños que nacen, el servicio de salud venezolano gasta alrededor de US\$ 7174 en costos directos médicos durante los primeros 5 años de vida a causa de esta enfermedad. Anualmente, en Venezuela se gastan cerca de US\$ 5 millones en el tratamiento hospitalario y ambulatorio de la gastroenteritis por rotavirus. Esta cifra es mayor que la notificada en Perú, donde los gastos médicos anuales ocasionados por esta enfermedad ascienden a aproximadamente US\$2,6 millones (23). Un programa de vacunación antirrotavírica en Venezuela disminuiría los costos en US\$2,5 millones.

En el análisis de costo-efectividad, se compara el costo neto de la aplicación de un programa de vacunación, o el costo del programa con lo que se ahorra por su aplicación, con la efectividad de dicho programa. A partir de los resultados del presente estudio, se calculó una relación costo-efectividad de US\$ 1371 por AVAD.

Para determinar si una intervención es costoefectiva se puede emplear el patrón de referencia de la OMS. En su Informe sobre la salud en el mundo de 2002, este organismo considera que una intervención médica es muy costoefectiva cuando la relación costoefectividad (US\$/AVAD) calculada es menor que el producto interno bruto (PIB) per cápita, y costoefectiva cuando dicha relación es equivalente a entre una y tres veces el valor del PIB per cápita (24). A partir de estos criterios, se considera que en Venezuela, la vacuna contra el rotavirus es muy costoefectiva, ya que la relación costoefectividad calculada es menor que el valor del PIB per cápita, evaluado en US\$ 3319 (12).

A la hora de interpretar los resultados obtenidos, surgen algunas limitaciones importantes del estudio. En ausencia de información sobre la mortalidad específica por rotavirus en Venezuela, se utilizaron los valores calcu- 
CUADRO 7. Análisis de sensibilidad de los costos médicos atribuidos al rotavirus y de la relación costo-efectividad de la vacunación antirrotavírica

\begin{tabular}{|c|c|c|c|c|}
\hline \multirow[b]{2}{*}{ Variable } & \multicolumn{2}{|c|}{ Costo médico por niño } & \multicolumn{2}{|c|}{ Costo-efectividad incremental } \\
\hline & $\begin{array}{l}\text { Valor }^{\mathrm{a}} \\
\text { (US\$) }\end{array}$ & $\begin{array}{l}\text { Variación } \\
(\%)\end{array}$ & $\begin{array}{c}\text { Valor a,b } \\
\text { (US\$/AVAD) }\end{array}$ & $\begin{array}{c}\text { Variación } \\
(\%)\end{array}$ \\
\hline \multicolumn{5}{|c|}{$\begin{array}{l}\text { Riesgo acumulado de hospitalización por rotavirus } \\
\text { (niños menores de } 5 \text { años) }\end{array}$} \\
\hline $0,019(-20 \%)$ & 6,66 & -7 & 1396 & +2 \\
\hline $0,023^{c}$ & 7,17 & - & 1371 & - \\
\hline $0,028(+20 \%)$ & 7,77 & +8 & 1342 & -2 \\
\hline \multicolumn{5}{|c|}{$\begin{array}{l}\text { Riesgo acumulado de consulta médica por rotavirus } \\
\text { (niños menores de } 5 \text { años) }\end{array}$} \\
\hline $0,152(-20 \%)$ & 6,31 & -12 & 1410 & +3 \\
\hline $0,190^{c}$ & 7,17 & - & 1371 & - \\
\hline $0,228(+20 \%)$ & 8,04 & +12 & 1331 & -3 \\
\hline \multicolumn{5}{|c|}{$\begin{array}{l}\text { Riesgo acumulado de muerte por rotavirus } \\
\text { (tasa por } 100000 \text { niños menores de } 5 \text { años) }\end{array}$} \\
\hline $49,6(-20 \%)$ & 7,17 & 0 & 1708 & +25 \\
\hline $62,0^{c}$ & 7,17 & - & 1371 & - \\
\hline $74,4(+20 \%)$ & 7,17 & 0 & 1145 & -16 \\
\hline \multicolumn{5}{|c|}{ Eficacia de la vacuna (muertes evitadas) } \\
\hline $0,70(-18 \%)$ & 7,17 & 0 & 1660 & $+21 \%$ \\
\hline $0,85^{\mathrm{c}}$ & 7,17 & - & 1371 & - \\
\hline $1,00(+18 \%)$ & 7,17 & 0 & 1167 & $-15 \%$ \\
\hline \multicolumn{5}{|c|}{ Eficacia de la vacuna (hospitalizaciones evitadas) } \\
\hline $0,70(-18 \%)$ & 7,17 & 0 & 1396 & +2 \\
\hline $0,85^{\mathrm{c}}$ & 7,17 & - & 1371 & - \\
\hline $1,00(+18 \%)$ & 7,17 & 0 & 1346 & -2 \\
\hline \multicolumn{5}{|l|}{ Costo medio diario/cama } \\
\hline US\$ 15,93 (-20\%) & 6,80 & -5 & 1389 & +1 \\
\hline US\$ $19,91^{\mathrm{c}}$ & 7,17 & - & 1371 & - \\
\hline US\$ 23,89 (+20\%) & 7,55 & +5 & 1352 & -1 \\
\hline \multicolumn{5}{|c|}{ Costo medio por consulta ambulatoria } \\
\hline US\$ $4,38(-20 \%)$ & 6,97 & -3 & 1380 & +1 \\
\hline US $\$ 5,48^{c}$ & 7,17 & - & 1371 & - \\
\hline US\$ $6,58(+20 \%)$ & 7,38 & +3 & 1361 & -1 \\
\hline \multicolumn{5}{|c|}{ Precio estimado de la vacuna (por régimen) } \\
\hline US\$ $16(-33 \%)$ & 7,17 & 0 & 822 & $-40 \%$ \\
\hline US\$ $24^{c}$ & 7,17 & - & 1371 & - \\
\hline US\$ 32 (+33\%) & 7,17 & 0 & 1919 & $+40 \%$ \\
\hline
\end{tabular}

lados por Parashar et al. y se hicieron algunas estimaciones. Los indicadores de morbilidad determinados en este estudio se basaron en información proveniente del Estado Carabobo, que presenta indicadores de salud por encima de la media del país, por lo que estas estimaciones están subestimadas para Venezuela. Por ejemplo, esta diferencia se observa cuando se comparan los datos epidemiológicos de la enfermedad por rotavirus de Valencia (1) con los de Caracas (3). En el caso de algunas pruebas de laboratorio y procedimientos complemen- tarios, no fue posible encontrar información sobre los costos, lo que condujo a hacer algunas estimaciones. En todos los casos, las estimaciones fueron conservadoras. No existen datos de efectividad de la vacuna contra el rotavirus, por lo que se trabajó sólo con datos de eficacia. Además, no se tuvo en cuenta en este análisis el impacto de la enfermedad por rotavirus en el hogar ni los costos derivados de la atención domiciliaria.

A pesar de estas limitaciones, el análisis realizado refleja de manera representativa el costo de la gastroenteritis por rotavirus y el beneficio por vacunación, a partir de información específica de Venezuela. Asimismo, el presente estudio crea oportunidades para comparar la relación de costoefectividad entre un programa de vacunación y otras medidas de control que sirvan como base para la toma de decisiones en materia de vacunación.

En conclusión, los resultados de este análisis de costo-efectividad indican que la aplicación de la vacuna antirrotavírica en la prevención de la gastroenteritis por rotavirus es una intervención eficiente con un costo razonable 
por cada AVAD evitado en niños venezolanos de hasta 5 años de edad.

Agradecimientos. Para la realización de este estudio se contó con el patrocinio de GlaxoSmithKline Biologicals. Los autores de este artículo agradecen a los Doctores José Levy, Yubizaly López, Olga Castillo de Febres, Marisol Escalona, Francisco Larrea y Mary Tomat, y a Belén Salinas, investigadora principal del estudio de vigilancia hospitalaria de la gastroenteritis por rotavirus, su contribución a la recopilación de datos indispensa-

\section{REFERENCIAS}

1. Salinas B, González G, González R, Escalona M, Materán M, Pérez-Schael I. Epidemiologic and clinical characteristics of rotavirus disease during five years of surveillance in Venezuela. Pediatr Infect Dis J. 2004;23(10): S161-7.

2. Parashar UD, Hummelman EG, Bresee IS, Miller MA, Glass RI. Global illness and deaths caused by rotavirus disease in children. Emerg Infect Dis. 2003;9(5):565-72.

3. Pérez-Schael I, González R, Fernández R, Alfonzo E, Inaty D, Boher Y, et al. Epidemiological features of rotavirus infection in Caracas, Venezuela: implications for rotavirus immunization programs. J Med Virol. 1999;59(4): 520-6.

4. Urrestarazu MI, Liprandi F, De Suárez EP, González R, Pérez-Schael I. Etiological, clini$\mathrm{cal}$, and sociodemographic characteristics of acute diarrhea in Venezuela. Pan Am J Public Health. 1999;6:149-56.

5. González FS, Sordo ME, Rowensztein G, Sabbag L, Roussos A, De Petre E, et al. Rotavirus diarrhea. Impact in a pediatric hospital of Buenos Aires. Medicina. 1999;59:321-6.

6. Villa S, Guiscafré $H$, Martínez $H$, Muñoz $\mathrm{O}$, Gutiérrez G. Seasonal diarrhoeal mortality among Mexican children. Bull World Health Organ. 1999;77(5):375-80.

7. Abate $\mathrm{H}$, Linhares $\mathrm{AC}$, Venegas $\mathrm{G}$, Vergara $\mathrm{R}$, López, P, Jiménez E, et al. Results of a hospital-based study of rotavirus gastroenteritis in Latin American children. Resumen presentado en el Congreso Internacional de Pediatría en Cancún, México. 15-20 de agosto de 2004.

8. O'Ryan M, Pérez-Schael I, Mamani N, Peña A, Salinas B, González G, et al. Rotavirusassociated medical visits and hospitalizations in South America: a prospective study at three large sentinel hospitals. Pediatr Infect Dis J. 2001;20(7):685-93.

9. Rheingans RD, Constenla D, Innis B, Breuer T, Antil L. Cost-effectiveness of vaccination for rotavirus gastroenteritis in Latin America. Rev Panam Salud Publica. (Aceptado para publicación).

10. Rheingans RD, Constenla D, Antil L, Innis B, Breuer T. Economic and health burden of rotavirus gastroenteritis in Latin America. Rev Panam Salud Publica. (Aceptado para publicación).

11. Pan-American Health Organization. Health Analysis and Information Systems Area. Regional Core Health Data Initiative; Technical Health Information System. Washington, D.C.; 2004.

12. World Bank. World Development Indicators on-line, 2004. [Sitio en Internet] El Banco Mundial. Hallado en: http://www.world bank.org/data/wdi2004/index.htm. Acceso el 12 de abril de 2005.

13. Venezuela, Ministerio de Sanidad y Departamento Social (MSDS), Dirección de Información Social y Estadística. Anuario de mortalidad 1998-2001 [Sitio en Internet] Ministerio de Sanidad y Departamento Social (MSDS). Hallado en: www. msds. gov.ve. Acceso el 15 de diciembre de 2004.

14. World Health Organization Statistical Information System (WHOSIS). Life tables for 191 countries. 2000. [Sitio en Internet] Hallado en: http://www3.who.int/whosis/menu.cfm? path=whosis, bod,burden statistics,life \& language=english. Acceso el 12 de abril de 2005.

15. Velázquez FR, Matson DO, Guerrero ML, Shults J, Calva JJ, Morrow AL, et al. Serum antibody as a marker of protection against natural rotavirus infection and disease. J Infect Dis. 2000;182(6):1602-9.

16. Murray CJL, López AD. The global burden of disease: a comprehensive assessment of mortality and disability from diseases, injuries and risk factors in 1990 and projected to 2020. Cambridge: Harvard University Press; 1996.

17. Baltussen R, Adam $T$, Tan Torres $T$, Hutubessy R, Acharya A, Evans D, et al. Generalized cost effectiveness analysis: a guide. Geneva: World Health Organization; 2002.

18. Liddle JL, Burgess MA, Gilbert GL, Hanson RM, McIntyre PB, Bishop RF, et al. Rotavirus gastroenteritis: impact on young children, bles para la realización de este estudio. También agradecen a las autoridades del Centro Docente La Trinidad de Caracas y del Hospital Metropolitano del Norte de Valencia por permitir el uso de la base de datos de sus departamentos de contabilidad. their families and the health care system. Med J Aust. 1997;167(6):304-7.

19. Ruiz-Palacios GM, Pérez-Schael I, Velázquez FR, Abate H, Breuer T, Clemens SC, et al. Safety and efficacy of an attenuated vaccine against severe rotavirus gastroenteritis. New Eng J Med. 2006 Jan 5; 354(1):11-22.

20. De Vos B, Vesikari T, Linhares AC, Salinas B, Pérez-Schael I, Ruiz-Palacios GM, et al. A rotavirus vaccine for prophylaxis of infants against rotavirus gastroenteritis. Ped Infect Dis J. 2004; 23:S179-S82.

21. World Health Organization, United Nations Children's Fund. WHO \& UNICEF estimates of national immunization coverage, 2003. [Sitio en Internet]. Hallado en: http:// www.who.int/vaccines surveillance/WHO UNICEF_Coverage_Review/. Acceso el 11 de octubre de 2004.

22. Venezuela, Instituto Nacional de Estadística, Ministerio de Sanidad y Departamento Social (MSDS), Dirección de Información Social y Estadística. Encuesta de hogares por muestreo, junio de 2003.

23. Ehrenkranz P, Lanata C F, Penny M E, Salazar-Lindo E, Glass R I. Rotavirus diarrhea disease burden in Peru: the need for a rotavirus vaccine and its potential cost savings. Rev Panam Salud Publica. 2001;10(4):240-8.

24. Organización Mundial de la Salud. Informe sobre la salud en el mundo 2002-Reducir los riesgos y promover una vida sana. [Sitio en Internet] Organización Mundial de la Salud (OMS). Disponible en: http://www.who.int/ whr/2002/es. Acceso el 12 de mayo de 2005.

Manuscrito recibido el 6 de septiembre de 2005. Aceptado para publicación, tras revisión, el 7 de junio de 2006 
ABSTRACT Objective. To assess the cost of medical care for rotavirus gastroenteritis and the cost-effectiveness of the antiretroviral vaccine in Venezuelan children under five.

\section{Assessment of the economic impact of the antiretroviral vaccine in Venezuela}

Key words
Methods. We used an economic model that comprises epidemiologic information, vaccine efficacy, and the cost of medical care in connection with rotavirus gastroenteritis, viewed from a social perspective. In order to determine the effectiveness of the vaccine, we estimated the number of hospitalized cases, of medical visits, and of deaths averted after vaccination. The cost-effectiveness of the vaccine was determined on the basis of the number of disability-adjusted life years (DALYs) and cases averted. Results. In Venezuela, health services spend approximately US\$ 4.2 million yearly on covering the costs of medical care for rotavirus-related disease. In a vaccinated cohort, an antiretroviral vaccination program would prevent around 52\% (186) of the deaths, 54\% (7 232) of the hospitalizations, and 50\% (55 168) of the ambulatory visits that take place during the first five years of life. For an estimated cost of approximately US\$ 24 per individual vaccination schedule, the cost-effectiveness ratio obtained is US\$ 1352 per DALY.

Conclusions. The results of this study suggest that antiretroviral vaccination is a cost-effective strategy for preventing rotavirus gastroenteritis in Venezuela, since it can prevent deaths and DALYs in the population under five years of age.

Rotavirus, vaccination, cost-effectiveness evaluation, prevention, gastroenteritis, Venezuela.

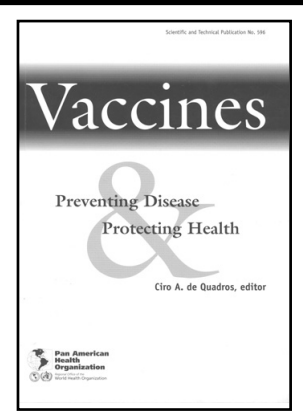

\section{Vaccines: Preventing Disease and Protecting Health IS NOW AVAILABLE IN ELECTRONIC FORMAT}

In this book you will find information about how vaccines have improved the health of the world's populations. The book relates successful efforts to fight disease with vaccines, including the eradication of polio from the Americas, and the potential contribution of new measles vaccine formulations to reducing measles mortality worldwide. It also looks at the challenges posed in using vaccines to cope with emerging and re-emerging diseases, such as HIV/AIDS, and in the fight against bioterrorism.

Electronic Version: 2004, 412p., ISBN 927511596 6, Order code: SP-E 596, Price: US $\$ 40.00$

To access the electronic version, follow these three simple steps:

1. Visit the site: www.ingentaselect.com

2. Select Pan American Health Organization in the Browse by publisher option

3. Select Vaccines: Preventing Disease and Protecting Health

Or go directly to PAHO publications by visiting:

http://www.ingentaselect.com/pubjournals.htm?pb=paho 